\title{
Closure of Emergency Midline Laparotomy over Subcutaneous Closed Suction Drain to Mitigate Incisional Surgical Site Infection, Dehiscence and Hernia: A Prospective Comparative Study Ashraf Mohammad El-Badry, Ahmed Gaber Mahmoud, Mohamed Mahmoud Ali ${ }^{*}$ \\ Department of Surgery, Faculty of Medicine, Sohag University, Sohag, Egypt
}

*Corresponding author: Mohamed Mahmoud Ali, Mobile: (+20)1004564240, E-mail: drmohamedmahmoud1969@gmail.com

\begin{abstract}
Background: The role of subcutaneous closed suction drainage (SCSD) during closure of emergency midline laparotomy (EML) for reduction of incisional surgical site infection (ISSI) remains debatable. Objective: The aim of the work was to investigate whether SCSD could minimize ISSI in the setting of emergency abdominal surgery. Patients and Methods: Adult patients with non-traumatic acute abdomen who underwent EML from June 2017 to January 2021 by single surgical team at Sohag University Hospital were prospectively enrolled. Patients were randomized according to EML incision closure technique into group A without SCSD and group B with closure of EML over SCSD. Both groups were compared regarding ISSI, wound dehiscence and incisional hernia. Results: Fifty-four patients were eligible (27 per group) with median age of 62 (range: 19-81) years. Both groups were comparable regarding gender and age. Group B exhibited significantly lower rates of ISSI (3 patients, 11\%) and wound dehiscence (zero) compared with group A, (12 patients, 44\%) and (5 patients, 15\%), respectively. Likewise, the duration of hospital stay was significantly shorter among patients in group B versus those in group A. Relaparotomy was required in 2 cases due to disruption of small bowel anastomosis in group A and leaking repair of duodenal ulcer in group B. After a median follow-up of 26 (range: 7 - 44) months, the protective effect of SCSD against ISSI correlated with significantly lower incidence of incisional hernia in group B (1 patient, 3.7\%) in comparison with group A (5 patients, 18.5\%). Conclusion: It could be concluded that mitigation of ISSI, wound dehiscence and incisional hernia with subcutaneous closed suction drainage favors its routine application during closure of non-traumatic EML.
\end{abstract}

Keywords: Laparotomy, Subcutaneous drainage, Surgical site infection

\section{INTRODUCTION}

Emergency midline laparotomy (EML) is fundamental to rescue emergency patients who suffer from non-traumatic acute abdomen ${ }^{(1-3)}$. Two forms of infections at the surgical site, including incisional surgical site infections (ISSI) and organ/space infections, are encountered in more than one third of patients following EML ${ }^{(4)}$. ISSI may hinder adjuvant therapy, trigger wound dehiscence and incisional hernia, prolong hospital stay and increase re-operations and mortality ${ }^{(5)}$. There is increased risk of ISSI with advanced age, lifestyle (smoking and alcohol intake), medications (steroids and immunosuppressive drugs) and associated co-morbidities (diabetes mellitus, hypertension, chronic obstructive pulmonary disease, renal insufficiency, etc) ${ }^{(6)}$.

Prophylaxis against ISSI comprises implementation of updated protocols for operative theaters' cleaning, sterilization of surgical instruments and maintenance of sterile barrier around the surgical field. ${ }^{7}$ Likewise, optimizing patient's specific risk factors, bowel preparation, skin decontamination, prophylactic antibiotics prior to skin incision and maintenance of normoglycemia should be accomplished before surgery ${ }^{(7,8)}$.

Intra-operatively, intra-peritoneal contamination can be diminished by copious wash-out of the peritoneal cavity and appropriate placement of drains (9). Irrigation and drainage of the subcutaneous tissues before wound closure by antiseptic solutions is also advocated ${ }^{(10-13)}$.

Closure of EML over subcutaneous closed suction drainage (SCSD) systems remains arguable ${ }^{\mathbf{1 4}-}$ ${ }^{16}$. Proponent of this strategy were able to demonstrate significant reduction of EML wound complications ${ }^{(\mathbf{1 6}}$, 17). However, other studies failed to show such beneficial effect ${ }^{(\mathbf{1 8}, \mathbf{1 9})}$.

The aim of the present study was to investigate whether SCSD could minimize ISSI in the setting of emergency abdominal surgery.

\section{PATIENTS AND METHODS}

This prospective study included a total of fifty-four patients with acute abdomen who underwent EML at Sohag University Hospital. This study was conducted between June 2017 to January 2021 .

Exclusion criteria: age < 18 years, trauma, bowel ischemia, laparotomy due to gynecologic, urologic, and vascular disorders and history of chemo- or radiotherapy within 6 months prior to presentation.

Patients were divided into 2 groups (27 in each group), group A (closure of EML without SCSD) versus group B (closure of EML over SCSD tube). Surgical site infection was defined according to the definitions of the Centers for Disease Control (CDC) and Prevention of the United States of America ${ }^{(20)}$, figure 1. 


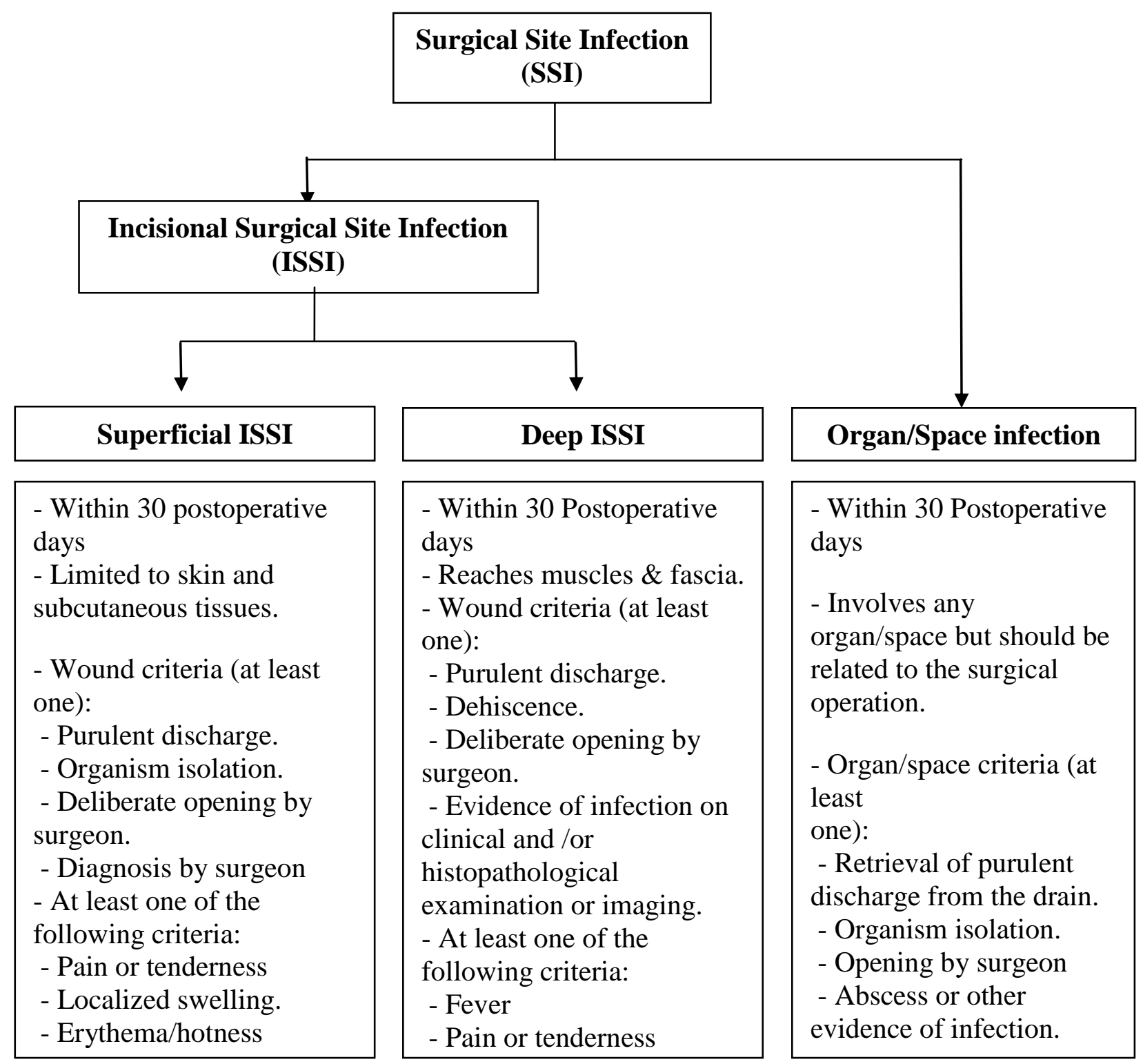

Figure (1): Types of Surgical Site Infection (SSI) according to definitions of the Centers for Disease Control and Prevention of the United States of America.

Our primary objective was to assess the influence of SCSD on incidence of ISSI. Moreover, the rates of wound dehiscence, organ/space infection and overall complications including incsional hernia were studied. Patients were randomly assigned in alternating manner to each group. Preoperatively, informed consent was signed by each patient.

Intra-operative measures to avoid surgical site infection:

All operative procedures were consistently performed by three consultant surgeons. A standardized protocol to reduce the rates of surgical site infection was applied in all patients. All patients received prophylactic antibiotic treatment during induction of anesthesia and another dose if surgery continued for more than 3 hours. Peritoneal irrigation/lavage was carried out using at least 4 liters of warm saline followed by appropriate placement of intra-abdominal drains. The EML wound was then irrigated with warm saline followed by povidone iodine.

After fascial closure using continuous nonabsorbable sutures, a fenestrated plastic catheter was placed in the subcutaneous space with separate exit from the EML wound. The external end of the subcutaneous drainage catheter was connected via a plastic tube to continuous negative suction plastic compressible container.

This SCSD system was maintained at least until the fifth postoperative day in all patients. Thereafter, the time of removal of the SCSD catheter was decided in accordance with the status of the wound and the amount and nature of the effluent fluid which was periodically retrieved after evacuation of the negative suction container.

\section{Ethical Consideration:}

An approval of the study was obtained from Sohag University academic and ethical committee. 
Every patient signed an informed written consent for acceptance of the operation. This work has been carried out in accordance with The Code of Ethics of the World Medical Association (Declaration of Helsinki) for studies involving humans.

\section{Statistical analysis}

Graphpad Prism 7 was used for statistical analysis. Quantitative data were demonstrated as median and range while number and percentages are used to express qualitative data. Quantitative variables were compared by Student t-test. Statistically significant difference between groups was concluded with $p$ value $<0.05$.

\section{RESULTS}

According to the study protocol, fifty-four patients were eligible (27 in each group). Demographics, clinical characteristics, and laboratory data were comparable in both groups. There were no significant differences regarding history of prior abdominal surgery, diabetes mellitus or hypertension. Preoperative clinical and laboratory data were listed in table 1.

The indications of emergency abdominal surgery via midline incision included digestive tract perforations due to complicated appendicitis, peptic ulcers, small bowel strangulation, colonic obstruction and gall bladder perforation following severe acute cholecystitis (figures 2, 3, 4 and 5). Other indications were iatrogenic biliary injuries during cholecystectomy and complicated acute pancreatitis. The number of cases according to the etiology of peritonitis was not significantly different between groups. Intraoperatively, there was no significant difference regarding operative time, blood loss and the amount of transfusions. These data, including surgical procedures, are shown in tables $\mathbf{2 , 3}$, and $\mathbf{4}$.

Overall, ISSI was documented in 15 out of 54 patients $(27.8 \%)$. The rate of ISSI was significantly lower among patients who were enrolled in group B (3/27 patients, $11 \%)$ versus group A (12/27 patients, $44 \%), \mathrm{p}<0.05$. The frequency of wound dehiscence of EML incision was zero in group B compared with 5/27 patients (15\%) among patients in group A, p <0.05.

The overall incidence of intraperitoneal (organ/space) infection was 9.3\% (5 patients including 2 in group A and 3 in group B). In group A, there was one case of leaking small bowel anastomosis that required relaparotomy and revision. The other was recurrent pancreatic abscess for which percutaneous drainage was carried out under guidance of computed tomography. In group B, there was one case of duodenal leak after repair of peptic ulcer which mandated relaparotomy for revision of duodenal repair. In addition, intraperitoneal abscess occurred in two cases who underwent appendectomy and peritoneal lavage due to ruptured acute appendicitis. Both cases resolved adequately by ultrasound-guided percutaneous drainage.

Patients enrolled in group B required significantly shorter period of hospital stay $\mathrm{p}<0.05$, likely due to the significantly decreased rates of ISSI. Overall, after a median follow-up of 26 (range: 7 - 44) months, incisional hernia developed in 6/54 (11\%) patients. However, the majority of cases was found in group A $(5 / 27$ patients, $18.5 \%)$, a rate that was significantly higher than group B $(1 / 27$ patients, $3.7 \%)$, p <0.05. Data on postoperative complications, hospital stay and the rate of incisional hernia were summarized in table 5.

Table (1): Demographics and preoperative clinical and laboratory data.

\begin{tabular}{|c|c|c|}
\hline & $\begin{array}{l}\text { Group A } \\
\text { No drain }\end{array}$ & $\begin{array}{c}\text { Group B } \\
\text { SCSD }\end{array}$ \\
\hline \multicolumn{3}{|c|}{ Demographics and clinical data* } \\
\hline - Male gender (n) & $18 / 27(67 \%)$ & $17 / 27(63 \%)$ \\
\hline - $\operatorname{Age}^{\S}$ (year) & $59(19-77)$ & $64(23-81)$ \\
\hline$-\mathrm{BMI}^{\S}\left(\mathrm{kg} / \mathrm{m}^{2}\right)$ & $29(24-35)$ & $27(22-31)$ \\
\hline \multicolumn{3}{|l|}{ - History of: } \\
\hline - Previous laparotomy & $6 / 27(22 \%)$ & $6 / 27(22 \%)$ \\
\hline - $\quad$ Smoking & $10 / 27(37 \%)$ & $11 / 27(41 \%)$ \\
\hline - Diabetes mellitus & $8 / 27(30 \%)$ & $8 / 27(30 \%)$ \\
\hline - Hypertension & $8 / 27(30 \%)$ & $9 / 27(33 \%)$ \\
\hline - Malignant disease & $4 / 27(15 \%)$ & $4 / 27(15 \%)$ \\
\hline \multicolumn{3}{|l|}{ Laboratory data $^{\S_{*}}$} \\
\hline - Hemoglobin (g/dl) & $12(11-15)$ & $13(11-14)$ \\
\hline - Albumin (g/dl) & $3.7(2.9-4.7)$ & $3.5(2.7-4.9)$ \\
\hline$-\mathrm{PC}(\%)$ & $75(66-98)$ & $77(69-106)$ \\
\hline
\end{tabular}


Table (2): Indications of emergency surgery per group.

\begin{tabular}{lcc}
\hline & $\begin{array}{c}\text { Group A } \\
\text { No drain }\end{array}$ & $\begin{array}{c}\text { Group B } \\
\text { SCSD }\end{array}$ \\
\hline Digestive tract perforation* & & \\
$-\quad$ Complicated appendicitis & $7 / 27(26 \%)$ & $8 / 27(29.6 \%)$ \\
$-\quad$ Peptic ulcer & $5 / 27(18.5 \%)$ & $6 / 27(22.2 \%)$ \\
$-\quad$ Small bowel strangulation: & & \\
$-\quad$ Strangulated hernia & $2 / 27(7.4 \%)$ & $3 / 27(11.1 \%)$ \\
$-\quad$ Bands/adhesions & $2 / 27(7.4 \%)$ & $2 / 27(7.4 \%)$ \\
$-\quad$ Colonic obstruction: & & \\
$-\quad$ Obstructive malignancy & $3 / 27(11.1 \%)$ & $2 / 27(7.4 \%)$ \\
$-\quad$ Volvulus & $1 / 27(3.7 \%)$ & $1 / 27(3.7 \%)$ \\
$-\quad$ Perforated gall bladder & $2 / 27(7.4 \%)$ & $2 / 27(7.7 \%)$ \\
Iatrogenic biliary injury* & $3 / 27(11.1 \%)$ & $2 / 27(7.4 \%)$ \\
Severe acute pancreatitis* & $2 / 27(7.4 \%)$ & \\
\hline non significant difference & & \\
\hline
\end{tabular}

Table (3): Surgical procedures

\begin{tabular}{|c|c|c|}
\hline Diagnosis & Surgical procedure $^{*}$ & $\mathbf{n}$ \\
\hline \multicolumn{3}{|l|}{ Digestive tract perforation } \\
\hline - Complicated appendicitis & Appendectomy & 15 \\
\hline - Peptic ulcer & Simple closure + omental patch & 11 \\
\hline \multirow{3}{*}{$\begin{array}{l}\text { - Small bowel strangulation: } \\
\text { - } \quad \text { Colonic obstruction: }\end{array}$} & Resection + primary anastomosis & 9 \\
\hline & & \\
\hline & Resection + Hartmann's procedure ${ }^{* *}$ & 2 \\
\hline \multirow[t]{2}{*}{ - Obstructive malignancy } & Resection + Hartmann's procedure ${ }^{\S}$ & 2 \\
\hline & Resection + primary anastomosis $\S^{\S}$ & 1 \\
\hline - Volvulus & Resection + Hartmann's peocedure & 2 \\
\hline - Perforated gall bladder & Cholecystectomy & 3 \\
\hline Iatrogenic biliary injury & Hepatico-jejunostomy (Roux-en-Y) & 5 \\
\hline Severe acute pancreatitis & Drainage of abscess \pm necrosectomy & 4 \\
\hline \multicolumn{2}{|l|}{ Total } & 54 \\
\hline \multicolumn{3}{|c|}{$\begin{array}{l}\text { Peritoneal lavage, intraperitoneal drainage and wound irrigation with povidone iodine were carried out in all } \\
\text { patients, }{ }^{* *} \text { obstructed recto-sigmoid cancer, }{ }^{\S} \text { Perforated left colon cancer, } \\
\$ \$ \text { Perforated appendicitis on top of cecal cancer (resection+ ileal-transverse anastomosis), } \\
\text { n; number }\end{array}$} \\
\hline
\end{tabular}

Table (4): Operative time, blood loss and transfusions

\begin{tabular}{lcc}
\hline & $\begin{array}{c}\text { Group A } \\
\text { No drain }\end{array}$ & $\begin{array}{c}\text { Group B } \\
\text { SCSD }\end{array}$ \\
\hline Operative time (m)* & $190(110-295)$ & $185(95-310)$ \\
Blood loss (ml)* & $550(300-950)$ & $600(250-1100)$ \\
Transfusions: & & \\
$-\quad$ Packed RBCs (unit)*§ & $1(0-3)$ & $1(0-4)$ \\
$-\quad$ FFP (units)*\$ & $2(0-5)$ & $3(0-7)$ \\
\hline
\end{tabular}

*non significant difference; § median (range); FFP, fresh frozen plasma; m, minute; ml, milliliter; RBCs, red blood cells 


\section{https://ejhm.journals.ekb.eg/}

Table (5): Postoperative wound complications, hospital stay and incisional hernia

\begin{tabular}{lccc}
\hline & Group A & Group B & p-value \\
\hline Nocisional surgical site infection (ISSI) & $12 / 27(44 \%)$ & $3 / 27(11 \%)$ & $<0.05^{*}$ \\
Wound dehiscence & $5 / 27(15 \%)$ & Zero & $<0.05^{*}$ \\
Duration of hospital stay (days) & $13(7-35)$ & $7(4-16)$ & $<0.05^{*}$ \\
Incisional hernia & $5 / 27(18.5 \%)$ & $1 / 27(3.7 \%)$ & $<0.05^{*}$ \\
\hline *significant difference & & & \\
\hline
\end{tabular}
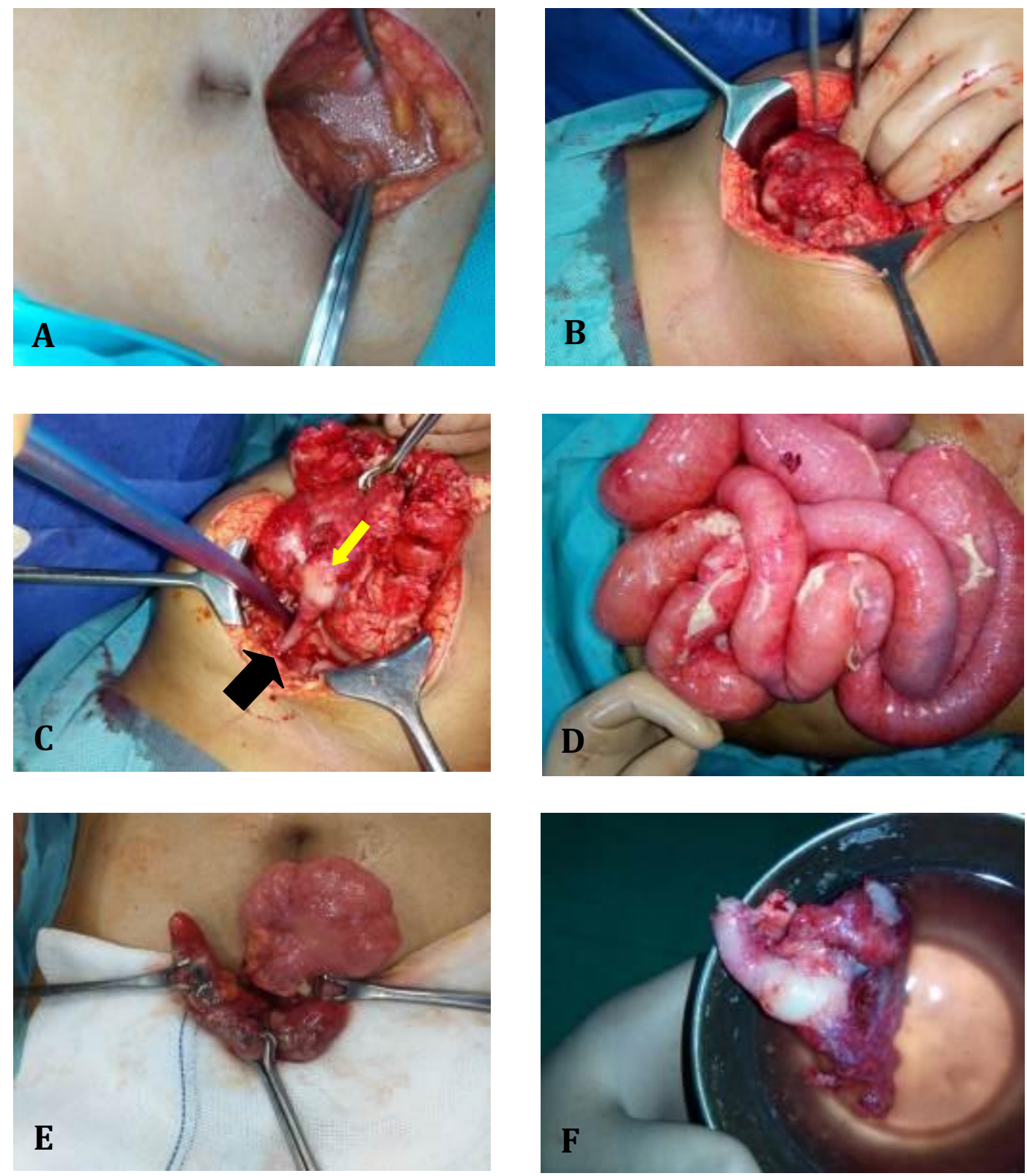

Figure (2): Peritonitis due to appendiceal perforation: A) Putulent fluid in the peritoneal cavity. B) Inflammatory mass around the cecum. C) Appendix identified (black arrow) with ischemic base (yellow arrow). D) Pyogenic membrane over small bowel surface. E) Appendix dissected off the cecum, periappendiceal phlegmon. F) Appendectomy specimen. 

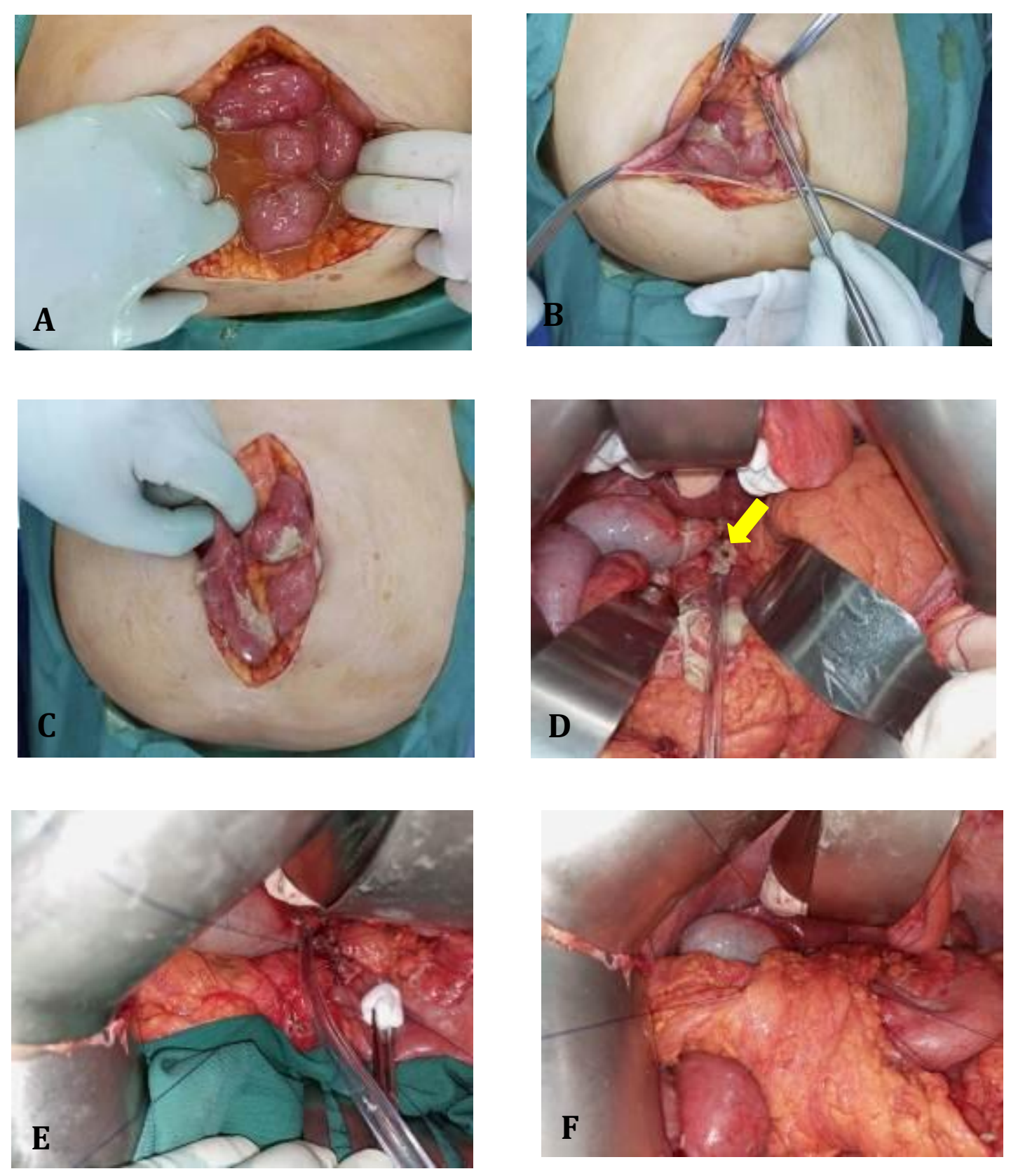

Figure (3): Peritonitis caused by perforated duodenal ulcer: A) Putulent intraperitoneal collection. B) Dissection of the small bowel from the anterior abdominal wall. C) Bowel surface covered with pyogenic membrane. D) Perforated duodenal ulcer (yellow arrow) seen after upward extension of EML wound. E) Repair of perforated duodenal ulcer with interrupted sutures. F) Omental flap fixation over duodenal ulcer repair. 

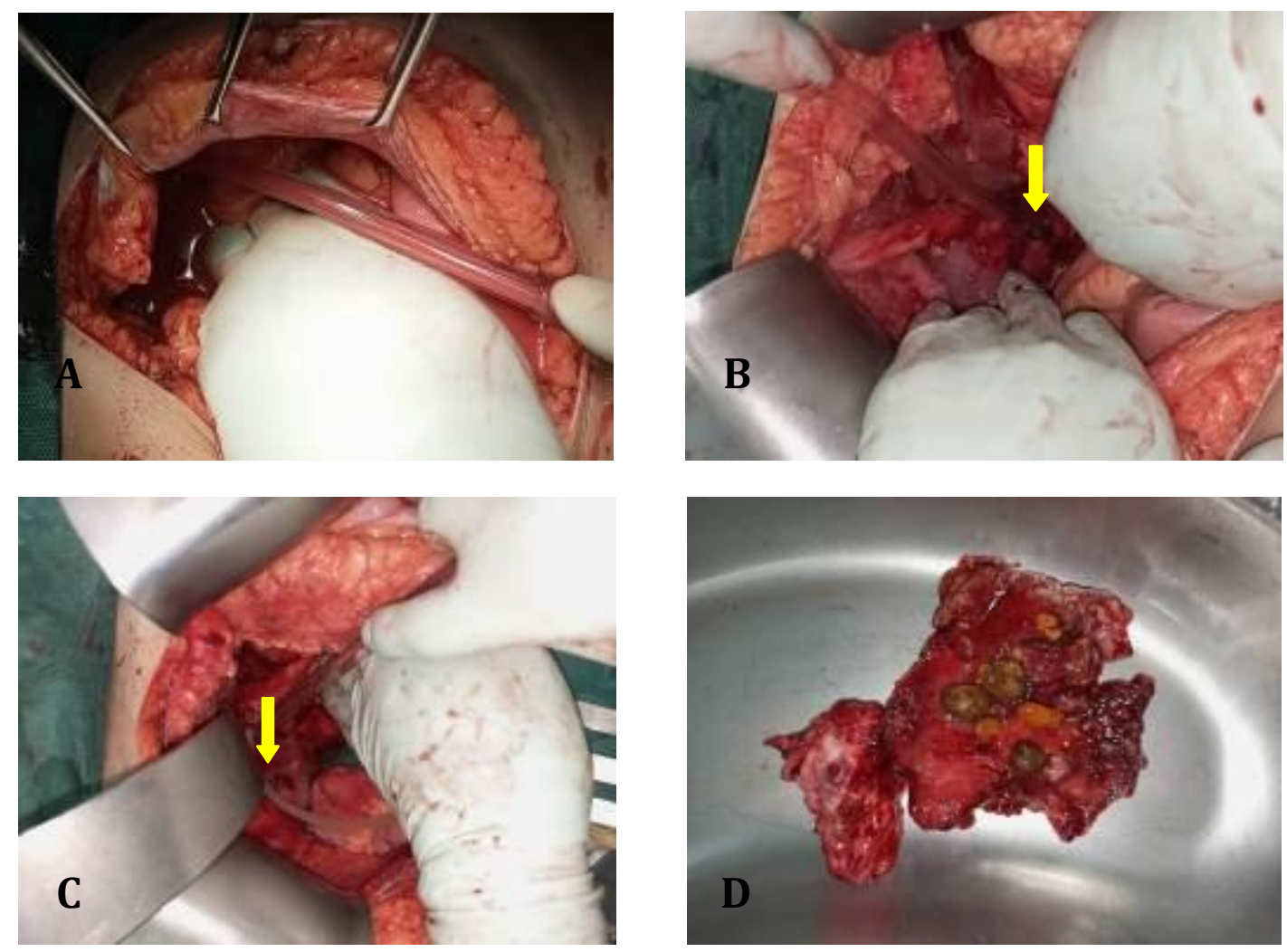

Figure (4): Peritonitis secondary to gall bladder rupture: A) Bile-stained intraperitoneal fluid. B) Stone (yellow arrow) emerging from inflammatory mass around ruptured gall bladder. C) Exploration of the common bile duct due to palpable stone inside and insertion of T-tube (yellow arrow). D) Ruptured gall bladder, excised specimen with stones.

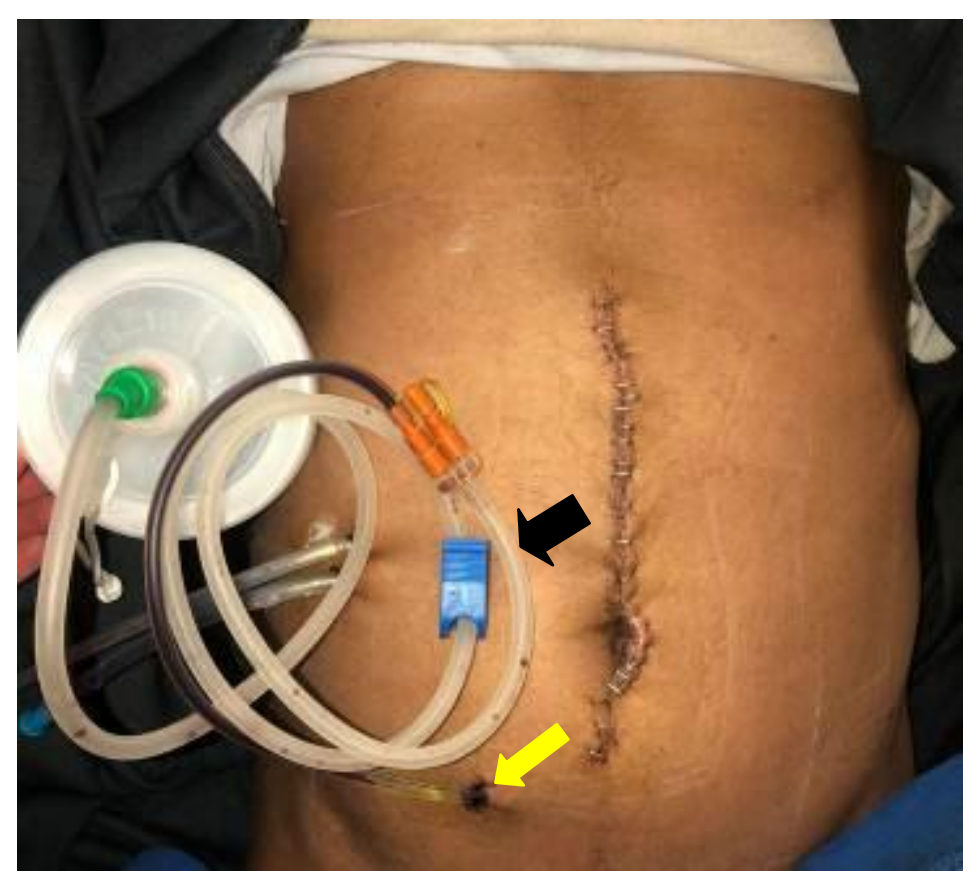

Figure (5): Subcutaneous Closed Suction Drainage (SCSD) device: Subcutaneous catheter with separate exit away from the midline laparotomy wound (yellow arrow). Plastic tube (black arrow) with valve (blue) connects the subcutaneous catheter with compressible plastic container (white). Compressing the plastic container followed by opening of the blue valve induces continuous subcutaneous suction and drainage under negative pressure. 


\section{DISCUSSION}

In this study we addressed the influence of SCSD during closure of midline laparotomy in the setting of emergency abdominal surgery. This approach correlated with significant reduction of ISSI, wound dehiscence, relaparotomies and increased rates of successful conservative management for intraperitoneal infection. Subsequently, the rates of incisional hernia were also significantly decreased.

Among patients who were enrolled in this study, $27.8 \%$ developed ISSI. The current literature shows wide range of variability regarding the rates of laparotomy related ISSI ${ }^{(\mathbf{2 1 - 2 4 )}}$. Thus, the rate of ISSI in our study was almost similar to study ${ }^{(25)}$ and relatively close to others which reported ISSI rate of $22.6 \%{ }^{(21)}$ and $21 \%{ }^{(22)}$. However, our results were obviously lower than Sumi et al. ${ }^{(23)}(36.2 \%)$ and higher than Zaidi and co-workers (13.8\%) ${ }^{(26)}$.

In our study, the distribution of patients with increased risk for ISSI due to advanced age, comorbidities, smoking and administration of drugs that may impair wound healing ${ }^{(6)}$ was homogeneous in both groups. Preoperative measures against ISSI ${ }^{\text {(7-10) }}$ were constantly applied in all patients. Moreover, all surgical procedures were performed by the same surgical team. Thus, the amelioration of ISSI rates in association with SCSD can be reasonably attributed to the benificial influence of subcutaneous suction drainage because the potential effect of other confounding factors was eliminated.

The currently available literature emphasized that a sufficient duration of SCSD after closure of emergency midline abdominal incision is crucial for avoidance of ISSI. For instance, Watanabi $\boldsymbol{e t}$ al. ${ }^{(5)}$ demonstrated that subcutaneous drainage of EML for a minimum of five days is associated with decreased rates of ISSI. Thus, the fifth postoperative day was set in our study as the erliest time point for removal of the subcutaneous suction drainage tube.

Several devices for continuous SCSD (a strategy known as negative pressure wound therapy) are available worldwide ${ }^{(17,24,27)}$. However, we could not use these devices due to their high cost, particularly in the setting of our limited resources. Therefore, we used a simple, relatively cost-effective and user-friendly system to achieve continuous SCSD of the subcutaneous space after EML.

Previous meta-analyses demonstrated conflicting results on the proposed protective effect of SCSD of laparotomy wounds against surgical site infection. For instance, a meta-analysis on patients pooled from nine studies showed that subcutaneous negative suction significantly reduces ISSI after laparotomy only in high risk patients like those with obesity and contaminated wound ${ }^{(\mathbf{1 9})}$. These results are supported by Coletta and his co-workers ${ }^{(18)}$ who failed to show beneficial effect of SCSD of clean-contaminated laparotomy wounds. However, another recent meta- analysis confirmed a significant reduction of surgical site infection by application of SCSD in 9 among 13 studies included in the analysis ${ }^{(28)}$. Of note, the controversy among these studies could be attributed to the heterogenous patient population, usage of different techniques of subcutaneous wound drainage and discrepancy of the duration before removal of the subcutaneous suction drains postoperatively.

Comparative randomized studies have convincingly shown that SCSD of laparotomy wounds resulted in remarkable reduction of wound seroma, infection and dehiscence ${ }^{(21)}$. Furthermore, the results of our study were in agreement with Sumi et al. ${ }^{(23)}$, who showed that following laparotomy for colorectal perforations, a significant decresae in the incidence of ISSI was accomplish in patients with SCSD of the laparotomy wound (16.7\%) compared with a rate of $56.5 \%$ in a control group without SCSD.

Despite the significant reduction of ISSI that we observed upon application of SCSD, there was no significant difference between both groups with regard to organ and intraperitoneal space infection. A similar result was also reported by a meta-analysis involving five studies on SSI after midline laparotomy ${ }^{(29)}$.

Incisional hernia following EML remains as surgical challenge. An Egyptian study on 80 patients who underwent EML due to digestive tract perforations, bowel ischemia, liver abscesses and pancreatitis, the incidence of incisional hernia was $25 \%{ }^{(30)}$. In another study, the rate of incisional hernia one year after EML which was carried out because of septic peritonitis was almost $17 \%{ }^{(\mathbf{3 1})}$. In our study, the overall occurrence of incisional hernia was $11 \%(6 / 54$ patients). This lower rate of incional hernia in our study compared with the available literature might be related to inclusion of patients other than those who had small bowel and colorectal pathology. These patients had peptic ulcer perforation, gall bladder rupture, acute pancreatitis and iatrogenic biliary injury. However, it should be noted that most of incisional hernia cases were found in group A (5/27 patients, $15 \%$ ), in which patients have not received SCSD versus only one case (3.7\%) in group B who received SCSD. This indicates that SCSD conferred significant protection against incisional hernia following EML.

The relatively small number of patients enrolled may represent a limitation in our study. However, it could be explained by inclusion of homogeneous study population (excluding trauma and pediatric patients) who were operated by the same surgical team.

\section{CONCLUSION}

This study demonstrated that closure of EML over subcutaneous closed suction drainage leads to significant reduction of ISSI and wound dehiscence, decreases hospital stay and treatment costs and protects against incisional hernia. 
Abbreviations: EML, emergency medline laparotomy; ISS, incisional surgical site infection; SCSD, subcutaneous closed suction drainage.

\section{Financial support and sponsorship: Nil.} Conflict of interest: Nil.

\section{REFERENCES}

1. Mc Geehan G, Edelduok I, Bucholc M et al. (2021): Systematic Review and Meta-Analysis of Wound Bundles in Emergency Midline Laparotomy Identifies That It Is Time for Improvement. Life (Basel), 11: 138-42.

2. Nally D, Sorensen J, Kavanagh D (2020): Emergency laparotomy research methodology: A systematic review. Surgeon, 18: 80-90.

3. Nally D, Sørensen J, Valentelyte G et al. (2019): Volume and in-hospital mortality after emergency abdominal surgery: a national population-based study. BMJ Open, 9: 32183-86.

4. Alkaaki A, Al-Radi O, Khoja A et al. (2019): Surgical site infection following abdominal surgery: a prospective cohort study. Can J Surg., 62: 111-117.

5. Watanabe J, Ota M, Kawamoto M et al. (2017): A randomized controlled trial of subcutaneous closed-suction Blake drains for the prevention of incisional surgical site infection after colorectal surgery. Int J Colorectal Dis., 32: 391-398.

6. Mueck K, Kao L (2017): Patients at High-Risk for Surgical Site Infection. Surg Infect (Larchmt), 18: 440446.

7. Weiser T, Forrester J, Forrester J (2019): Tactics to Prevent Intra-Abdominal Infections in General Surgery. Surg Infect (Larchmt), 20: 139-145.

8. Ohge H, Mayumi T, Haji $S$ et al. (2021): The Japan Society for Surgical Infection: guidelines for the prevention, detection, and management of gastroenterological surgical site infection, 2018. Surg Today, 51: 1-31.

9. Norman G, Goh E, Dumville J et al. (2020): Negative pressure wound therapy for surgical wounds healing by primary closure. https://pubmed.ncbi.nlm.nih. gov/32356396/

10. Papadakis M (2021): Wound irrigation for preventing surgical site infections. World J Methodol., 11: 222-227.

11. De Simone B, Sartelli M, Coccolini F et al. (2020): Intraoperative surgical site infection control and prevention: a position paper and future addendum to WSES intra-abdominal infections guidelines. World $\mathrm{J}$ Emerg Surg., 15: 10-13.

12. Ambe $\mathbf{P}$, Rombey $\mathrm{T}$, Rembe $\mathrm{J}$ et al. (2020): The role of saline irrigation prior to wound closure in the reduction of surgical site infection: a systematic review and metaanalysis. Patient Saf Surg., 14: 47-51.

13. de Jonge $\mathrm{S}$, Boldingh Q, Solomkin $\mathrm{J}$ et al. (2017): Systematic Review and Meta-Analysis of Randomized Controlled Trials Evaluating Prophylactic Intra-Operative Wound Irrigation for the Prevention of Surgical Site Infections. Surg Infect (Larchmt), 18: 508-519.

14. Seaman A, Sarac B, ElHawary $\mathrm{H}$ et al. (2021): The effect of negative pressure wound therapy on surgical site occurrences in closed incision abdominal wall reconstructions: a retrospective single surgeon and institution study. Hernia, 25: 1549-1555.
15. Gejoe G, Yadev I, Rahul M (2017): Emergency Laparotomies at a Tertiary Care Center-a Hospital-Based Cross-Sectional Study. Indian J Surg., 79: 206-211.

16. Cheong Chung J, Ali O, Hawthornthwaite $\mathbf{E}$ et al. (2021): Closed incision negative pressure wound therapy is associated with reduced surgical site infection after emergency laparotomy: A propensity matched-cohort analysis. Surgery, 170: 1568-1573.

17. Sahebally S, McKevitt K, Stephens I et al. (2018): Negative Pressure Wound Therapy for Closed Laparotomy Incisions in General and Colorectal Surgery: A Systematic Review and Meta-analysis. JAMA Surg., 153: 1834-38.

18. Coletta D, Del Basso C, Giuliani G et al. (2019): Subcutaneous suction drains do not prevent surgical site infections in clean-contaminated abdominal surgery-results of a systematic review and meta-analysis. Langenbecks Arch Surg., 404: 663-668.

19. Manzoor B, Heywood N, Sharma A (2015): Review of Subcutaneous Wound Drainage in Reducing Surgical Site Infections after Laparotomy. Surg Res Pract., 15: 715803. https://pubmed.ncbi.nlm.nih.gov/26783556/

20. Zabaglo M, Sharman T (2021): Postoperative Wound Infection. StatPearls. Treasure Island, FL. StatPearls Publishing. https://www.ncbi.nlm.nih.gov/books/NBK560533/

21. Wani J, Bhat J (2019): Role of Negative Suction Subcutaneous Drains in Contaminated Abdominal Surgeries. J Gastrointest Dig Syst., 9: 587-90.

22. Khan A, Mahesh K (2016): Role of Negative Suction Drain in Subcutaneous Plane in Reducing Laprotomy Wound Infection. IJSR., 5: 692-694.

23. Sumi Y, Yamashita K, Kanemitsu K et al. (2014): Effects of Subcutaneous Closed Suction Drain for the Prevention of Incisional SSI in Patients with Colorectal Perforation. Surgical Science, 5: 122-127.

24. Alsafrani T, Alabbasi A, Dabroom A et al. (2021): The Effectiveness of Superficial Drain to Reduce Surgical Site Infection in Colorectal Surgery. Cureus, 13: 17232-17235.

25. Hassan R, Osman S, Aabdeen $M$ et al. (2020): Incidence and root causes of surgical site infections after gastrointestinal surgery at a public teaching hospital in Sudan. Patient Saf Surg., 14: 45-49.

26. Zaidi A, El-Masry S (2017): Closed-incision negativepressure therapy in high-risk general surgery patients following laparotomy: a retrospective study. Colorectal Dis., 19: 283-287.

27. Boland P, Kelly M, Donlon N et al. (2021): Prophylactic negative pressure wound therapy for closed laparotomy wounds: A systematic review and meta-analysis of randomised controlled trials. Ir J Med Sci., 190: 261-267.

28. Fowler A, Barry M (2020): Closed incision negative pressure therapy: Results of recent trials and recommendations for clinical practice. Surgeon, 18: 241250.

29. Wells C, Ratnayake C, Perrin J et al. (2019): Prophylactic Negative Pressure Wound Therapy in Closed Abdominal Incisions: A Meta-analysis of Randomised Controlled Trials. World J Surg., 43: 2779-2788.

30. Saleem A, Abdallah H, Abdul Raheem $O$ et al. (2016): Rate of development of incisional hernia 1 year after urgent midline laparotomy. Al-Azhar Assiut Med J., 14: 59-66.

31. Mir S, Akram W, Gilkar I et al. (2020): Short Term Incidence of Incisional Hernia after Emergency Midline Laparotomy. Journal of Evidence Based Medicine and Healthcare, 7: 591-595. 